\title{
Restricted Aggregate Formation on Tetraphenylethene-Substituted Polythiophenes
}

\author{
João Pina,* Ana Clara B. Rodrigues, Mohamed Alnady, Wenyue Dong, Ullrich Scherf, \\ and J. Sérgio Seixas de Melo
}

Cite This: J. Phys. Chem. C 2020, 124, 13956-13965

Read Online

\section{ACCESS}

Џll Metrics \& More

Article Recommendations

Supporting Information

ABSTRACT: Two new polythiophenes functionalized with different degrees of tetraphenylethene, TPE, side groups (with TPE moieties at each thiophene unit, homoPT, and one in which only each second thiophene unit carries a TPE side chain, coPT) were successfully synthesized and characterized together with a poly(3hexylthiophene) with similar average molecular weight and a tetraphenylethene-thiophene model compound. The study aimed to understand the role of the sterically bulky TPE pendant groups on the inhibition of intra- and/or interchain packing (aggregation) of these conjugated polymers. For the tetraphenylethenethiophene model, compound aggregation induced emission (AIE) is active for water fractions $\geq 90 \%$. An opposite behavior

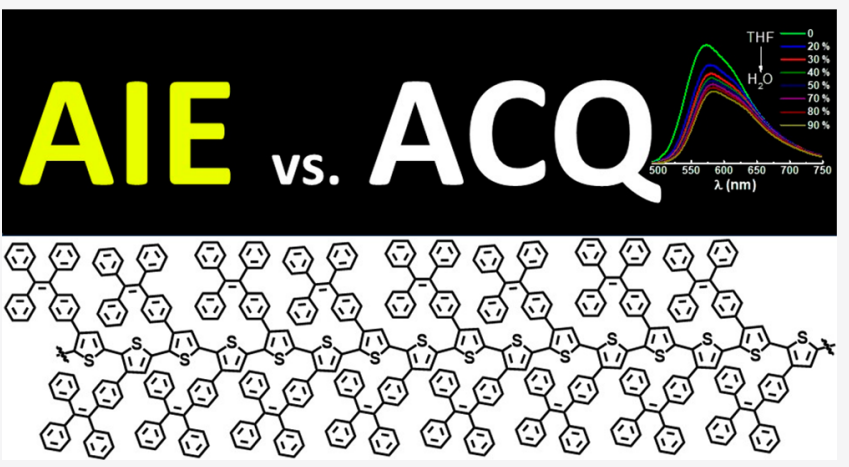
is found for the polythiophenes (homoPT and coPT) where aggregation caused quenching (ACQ) was found to occur both in solution and in the solid state. For the polythiophene with the higher degree of TPE labeling, homoPT, the amount of ACQ is significantly decreased. This is attributed to the high TPE substitution density in the polymer which promotes a more twisted conformation of the polymer backbone as the emissive chromophore. For the TPE-polythiophenes, only the energy migration mechanism was found to be active since the bulky TPE substituents restrict the polymer's intramolecular motions. The experimental observations of a small shift in the emission spectra of homoPT and significant hindering of ACQ on going from solution to the solid state explain the absence of intermolecular interactions together with the restriction of intramolecular rotations.

\section{INTRODUCTION}

Polythiophenes are among the most studied conjugated polymers due to their applications in the last generation of optoelectronic devices, namely, in organic solar cells, lightemitting diodes, and field effect transistors. ${ }^{1,2}$ It is increasingly clear that the electronic properties of conjugated polymers are highly dependent on the physical conformation of the polymer chains and the way the chains pack together in films. An obstacle to their development is the significant fluorescence quenching in the solid state that generally occurs due to strong interchain interaction of the polythiophenes. ${ }^{3}$ This phenomenon can be referred to as aggregation-caused quenching (ACQ). This may dramatically affect the performance of the polymers for optoelectronics because they are commonly used as solid films in their practical applications. Several chemical, physical and engineering strategies have been developed to overcome the ACQ effect, for example, attachment of bulky alicyclics, encapsulation by amphiphilic surfactants and blending with transparent polymers. ${ }^{4,5}$ These processes are, however, often accompanied by severe side effects. For example, the steric effects of bulky alicyclics can twist the conformations of the chromophoric units and hinder the electronic conjugation on the polymers, while the electronic effects of the saturated surfactants and nonconjugated polymers can dilute the chromophoric units densities and block the charge transport in electroluminescence (EL) devices.

Construction of a large variety of aggregation induced emission (AIE) polymers has been reported with various synthetic strategies to incorporate typical AIE active molecules (AIEgens), such as the propelled shaped molecules TPE and hexaphenylsilole, into polymer structures either by directly using AIEgen-containing monomers for homopolymerization or copolymerization or by modifying polymers with AIEgens through postpolymerization modification techniques. ${ }^{6-8}$ In this work, an attempt was made to circumvent the ACQ effect by synthesizing two tetraphenylethene-polythiophenes with

Received: November 21, 2019

Revised: March 17, 2020

Published: May 26, 2020 
Scheme 1. Synthetic Route for the TPE Boronic Ester 3

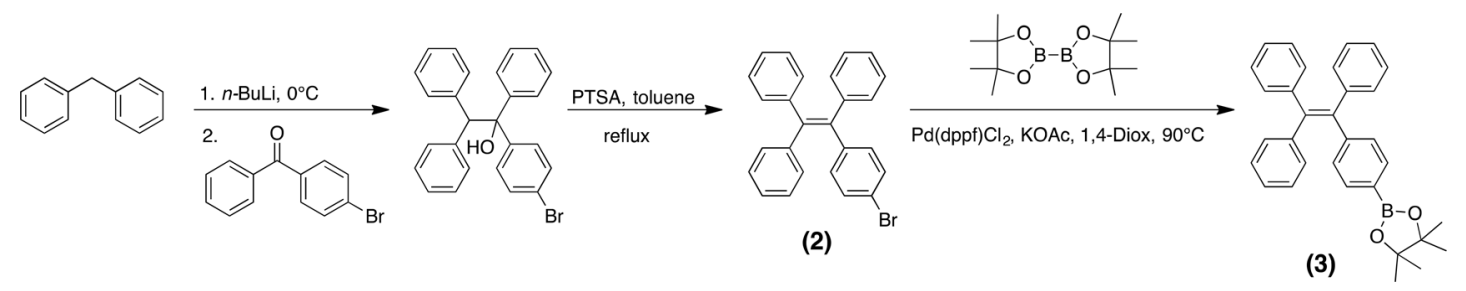

Scheme 2. Synthetic Route to Monomer 4 and the Corresponding Polymers HomoPT and CoPT<smiles>CC1CCB(c2ccc(C(=C(c3ccccc3)c3ccccc3)c3ccccc3)cc2)O1</smiles>

(3)

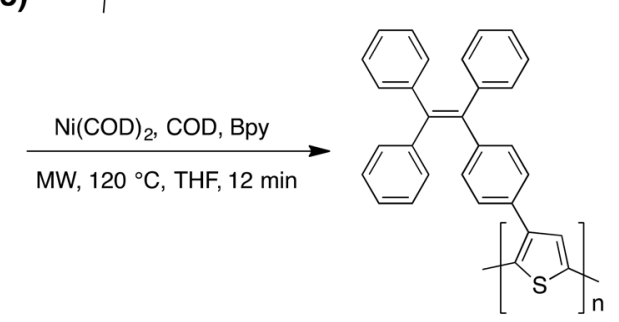

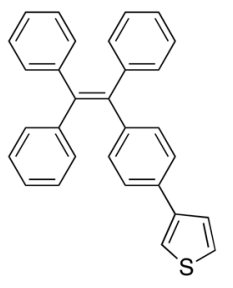

S

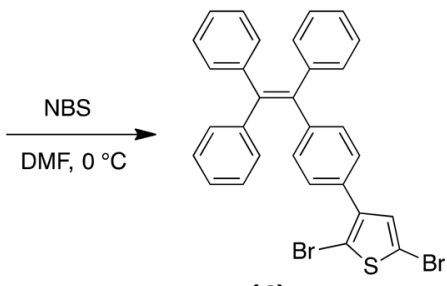

(4)

homoPT

(4)
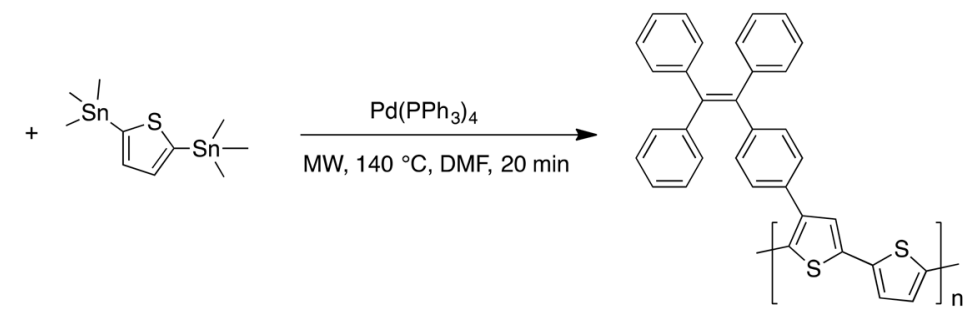

coPT

different degrees of substitution. The TPE molecule has been at the forefront of the development of the concept of AIE in which molecules that display very weak fluorescence in dilute solutions (of good solvents) become highly emissive upon aggregation in poor solvents or cast into solid films. ${ }^{9}$ Indeed, it has been demonstrated that TPE with its propeller-shaped conformation is an AIE active chromophore. ${ }^{9,10}$ The AIE effect is caused by the restriction of intramolecular rotations (RIR), associated with the loose bolt effect for the radiationless transitions, ${ }^{11}$ by aggregate formation, which effectively blocks the nonradiative energy dissipation channels (particularly the internal conversion) in favor of the radiative decay channel. In the aggregates, the propeller-shaped TPE units prevent the molecules from packing in a $\pi-\pi$ stacking process, while their intramolecular rotations are physically constrained in the solid state. $^{12}$

\section{EXPERIMENTAL SECTION}

Spectroscopic Measurements. All the solvents used in the photophysical experiments were of spectroscopic grade. Absorption and fluorescence spectra were recorded on Shimadzu UV-2100 (or Cary 5000 UV-vis-NIR) and Horiba-Jobin-Ivon SPEX Fluorog 3-22 (or Fluoromax) spectrometers, respectively. The fluorescence spectra were corrected for the wavelength response of the system. For the steady state and time-resolved emission experiments, the absorption at the excitation wavelength was kept below 0.1 . The fluorescence quantum yields were measured using either one of two methods: (i) the comparative method using as standards quinine sulfate (in $0.5 \mathrm{M} \mathrm{H}_{2} \mathrm{SO}_{4}$ solution, $\phi_{\mathrm{F}}=$ $0.546^{13}$ ) and quinquethiophene (dioxane solution, $\phi_{\mathrm{F}}=$ $0.36^{14}$ ) or (ii) the absolute method with a Hamamatsu Quantaurus QY absolute photoluminescence quantum yield spectrometer model C11347 (integrating sphere). ${ }^{8}$

Thin films from the compounds were obtained with a desktop precision spin-coating system, model P6700 series from Speedline Technologies. Solid-state thin films from the samples were obtained by deposition of a few drops from a solution of the compounds into a circular sapphire substrate (10 mm diameter) followed by spin-coating $(2500 \mathrm{rpm})$ in a nitrogen-saturated atmosphere ( $2 \mathrm{psi})$. The solutions for spincoating were prepared by adding $2 \mathrm{mg}$ of the samples to 200 $\mu \mathrm{L}$ of chloroform solution with stirring at $40{ }^{\circ} \mathrm{C}$ for $30 \mathrm{~min}$. In the solid state (thin films) the fluorescence quantum yields were measured using the absolute method with a Hamamatsu 
Scheme 3. Structures and Acronyms of the Investigated Compounds

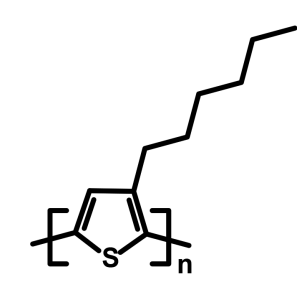

P3HT

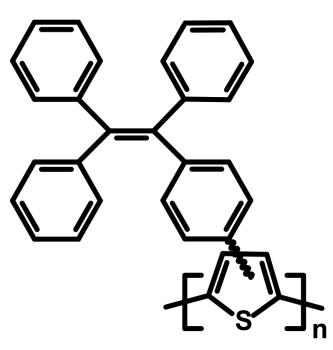

homoPT

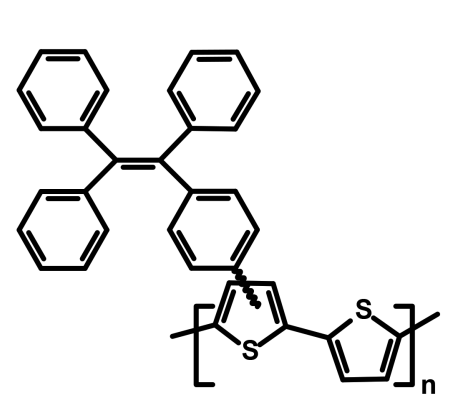

coPT

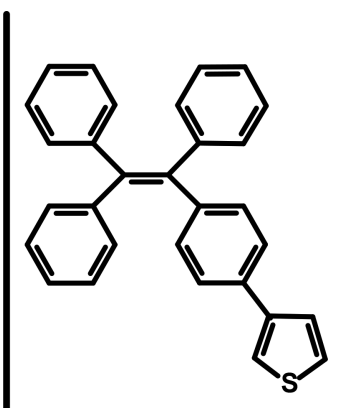

T-TPE
Quantaurus QY absolute photoluminescence quantum yield spectrometer, model C11347 (integration sphere). ${ }^{8}$

Fluorescence decays were measured using a home-built TCSPC system described elsewhere. ${ }^{15}$ Alternate measurements of the pulse profile at the excitation wavelength and the sample emission were performed until $5 \times 10^{3}$ counts maximum were reached. The fluorescence decays were analyzed using the modulating functions method of Striker with automatic correction for the photomultiplier "wavelength shift." 16 Temperature control was achieved using a home-built system based on cooled nitrogen and electric heating.

Singlet oxygen sensitization quantum yields were obtained by comparison of the measured singlet oxygen phosphorescence signal at $1275 \mathrm{~nm}$ followed upon irradiation of the aerated toluene solutions of the samples and reference compound, collected with excitation at $355 \mathrm{~nm}$ from a Nd:YAG laser with a setup elsewhere described. ${ }^{17}$ Perinaphthenone in toluene $\left(\phi_{\Delta}=0.93\right)$ was used as standard. ${ }^{18}$

Synthesis and Characterization. Regioregular poly(3dodecylthiophene-2,5-diyl), average $\bar{M}_{\mathrm{w}} \sim 60000 \mathrm{~g} \mathrm{~mol}^{-1}$, was purchased from Sigma-Aldrich and used as received without further purification. Poly(3-hexylthiophene), P3HT, with $\bar{M}_{\mathrm{w}}$ of $18500 \mathrm{~g} \mathrm{~mol}^{-1}$ was synthesized by conventional methods and analytically characterized as elsewhere described. ${ }^{19,20}$ Scheme 1 presents the synthetic route for the TPE boronic ester precursor. Monobromo-TPE 2 was obtained by treating 4-bromobenzophenone with diphenylmethyl lithium followed by acid-catalyzed dehydration. The TPE boronic ester 3 was synthesized by Suzuki-Miyaura-type coupling of monobromoTPE 2 and bis(pinacolato)diboron.

The synthesis route to monomer 4 and the corresponding polymers homoPT and coPT is depicted in Scheme 2. TPEsubstituted thiophene was obtained in a Suzuki-type aryl-aryl crosscoupling between 3-bromothiophene and TPE boronic ester. The TPE-substituted dibromothiophene monomer $\mathbf{4}$ was synthesized by bromination with NBS at $0{ }^{\circ} \mathrm{C}$. 2,5Bis(trimethylstannyl)thiophene was purchased from SigmaAldrich and used without further purification. Polymer homoPT was generated in a Yamamoto-type homocoupling with the mixture of $\mathrm{Ni}(\mathrm{COD})_{2}, 1,5$-cyclooctadiene (COD), 2,2'-bipyridine (Bpy), and THF under microwave (MW) heating at $120{ }^{\circ} \mathrm{C}$ for $12 \mathrm{~min}$. Polymer coPT was made in a Stille-type cross-coupling with the mixture of $\mathrm{Pd}\left(\mathrm{PPh}_{3}\right)_{4}$ and DMF under MW heating at $140{ }^{\circ} \mathrm{C}$ for $20 \mathrm{~min}$. The structure elucidation of monomer 4 was performed by NMR spectroscopy, mass spectrometry, and elemental analysis. The chemical structure of the obtained polymers was finally confirmed by NMR spectroscopy, GPC, and optical spectroscopy.
Polymer HomoPT. A mixture of monomer $4(400 \mathrm{mg}$, $0.699 \mathrm{mmol}), \mathrm{Ni}(\mathrm{COD})_{2}$ (500 mg, $\left.1.817 \mathrm{mmol}\right)$, BРy (284 $\mathrm{mg}, 1.817 \mathrm{mmol}$ ), and COD (197 mg, $1.817 \mathrm{mmol}$ ) in THF (6 $\mathrm{mL}$ ) was reacted under microwave heating at $120{ }^{\circ} \mathrm{C}$ for 12 min. The reaction mixture was quenched with water and extracted with chloroform. The collected organic phases were washed with aqueous $2 \mathrm{M} \mathrm{HCl}$ solution, aqueous $\mathrm{NaHCO}_{3}$ solution, saturated, aqueous EDTA solution, and brine and finally dried over $\mathrm{MgSO}_{4}$. Afterward, the solvents were removed under vacuum. The resulting solid was dissolved in a small amount of chloroform and precipitated into methanol $(500 \mathrm{~mL})$ to afford the target orange-colored polymer. Subsequent Soxhlet extractions were carried out with methanol, acetone, ethyl acetate, and chloroform. The orange polymer was obtained with $52 \%$ yield from the chloroform fraction $(150 \mathrm{mg}) .{ }^{1} \mathrm{H}$ NMR $\left(600 \mathrm{MHz}, \mathrm{C}_{2} \mathrm{D}_{2} \mathrm{Cl}_{4}, 60{ }^{\circ} \mathrm{C}\right) \delta$ (ppm) 7.26-6.60 (m, 20H). $\bar{M}_{\mathrm{n}} 14600, \bar{M}_{\mathrm{w}} 57800, \bar{M}_{\mathrm{w}} / \bar{M}_{\mathrm{n}}$ 3.96 (GPC, PS calibration).

Polymer CoPT. Monomer 4 (400 mg, $0.699 \mathrm{mmol})$, 2,5bis(trimethylstannyl)thiophene (286 $\mathrm{mg}, 0.699 \mathrm{mmol})$, and $\mathrm{Pd}(\mathrm{PPh} 3) 4$ (40.4 mg, $0.035 \mathrm{mmol}$ ) were added into a $20 \mathrm{~mL}$ microwave tube. The mixture was carefully degassed under vacuum for $15 \mathrm{~min}$. Next, dry DMF $(10 \mathrm{~mL})$ was added into the tube under argon and the resulting mixture was heated to $140{ }^{\circ} \mathrm{C}$ under microwave conditions for $20 \mathrm{~min}$. After that, 2(tributylstannyl)thiophene $(5.2 \mathrm{mg}, 0.014 \mathrm{mmol}$ ) was added as the end-capping agent, and the mixture was reacted for another $5 \mathrm{~min}$. The mixture was cooled down to room temperature, treated with aqueous $2 \mathrm{M} \mathrm{HCl}$ solution, and extracted with chloroform three times. The organic phases were collected and dried over $\mathrm{MgSO}_{4}$. Afterward, the solvents were removed under vacuum. The resulting solid was dissolved in a small amount of chloroform and precipitated into methanol (500 $\mathrm{mL}$ ) to afford the target polymer as a dark red solid. Subsequent Soxhlet extractions were carried out with methanol, acetone, ethyl acetate, and chloroform. After reprecipitation of the chloroform-soluble fraction into methanol, the dark red polymer was obtained with $69 \%$ yield (240 mg). ${ }^{1} \mathrm{H}$ NMR $\left(600 \mathrm{MHz}, \mathrm{C}_{2} \mathrm{D}_{2} \mathrm{Cl}_{4}, 60{ }^{\circ} \mathrm{C}\right) \delta(\mathrm{ppm})$ 7.49-6.59 (m, 22H). $\bar{M}_{\mathrm{n}} 14500, \bar{M}_{\mathrm{w}} 76600, \bar{M}_{\mathrm{w}} / \bar{M}_{\mathrm{n}} 5.28$ (GPC, PS calibration).

Instrumentation for Analytical Characterization. NMR spectra were recorded on a Bruker AVANCE 400 or AVANCE III 600 instrument. ${ }^{1} \mathrm{H}$ NMR and ${ }^{13} \mathrm{C}$ NMR spectra were measured with tetramethylsilane (TMS) as the internal standard. Gel permeation chromatography (GPC) measurements at a high temperature $\left(135^{\circ} \mathrm{C}\right)$ were carried out on a Waters Alliance 2000 System equipped with a PLgel-Guard 
and PLgel-MIXED-B-column (both from Agilent Technologies) using 1,3,5-trichlorobenzene as the eluent, with a RI detector and measured against polystyrene. Atmospheric pressure laser ionization (APLI) measurements were carried out on Bruker Daltronik Bremen with micrOTOF.

Elemental analyses were performed on a Vario EL II (CHNS) instrument.

\section{RESULTS AND DISCUSSION}

The structures and acronyms of the investigated compounds are depicted in Scheme 3. The study is focused to the spectroscopic investigation of polythiophenes comprising tetraphenylethene substituents, in each repeat unit, homoPT, or each second repeat unit, coPT. In addition, for comparison purposes, also poly(3-hexylthiophene), P3HT (displaying a similar degree of polymerization, DP), together with the monomeric model compound tetraphenylethene-thiophene, TTPE, were studied. The average molecular weight, $\bar{M}_{\mathrm{n}}$, weightaverage molecular weight, $\bar{M}_{\mathrm{w}}$, and polydispersity (PD) of the investigated polymers are presented in Table 1. On the basis of the $\bar{M}_{\mathrm{w}}$ data, the degree of polymerization (DP) varies between 110 and 154 (Table 1).

Table 1. Physical Characteristics for the Investigated Polythiophenes $^{a}$

\begin{tabular}{lcccc}
\multicolumn{1}{c}{ compd } & $\bar{M}_{\mathrm{n}}\left(\mathrm{g} \mathrm{mol}^{-1}\right)$ & $\bar{M}_{\mathrm{w}}\left(\mathrm{g} \mathrm{mol}^{-1}\right)$ & PD & DP \\
P3HT & 14000 & 18500 & 1.32 & 110 \\
homoPT & 14500 & 57800 & 3.96 & 140 \\
coPT & 14500 & 76600 & 5.28 & 154
\end{tabular}

${ }^{a}$ Mean and weight-average molecular weights $\left(\bar{M}_{\mathrm{n}}, \bar{M}_{\mathrm{w}}\right)$, polydispersity (PD), and degree of polymerization (DP) as calculated from $\bar{M}_{\mathrm{w}}$.

Spectroscopic and Photophysical Data. Absorption and fluorescence emission spectra of the investigated polythiophenes in toluene solution and in the solid state (thin films) are shown in Figure 1. The spectroscopic data for homoPT, coPT, and P3HT in solution displays the characteristic low energy unstructured absorption band together with the structured fluorescence emission band (clear structuring observed for coPT and P3HT, only allusively for homoPT)

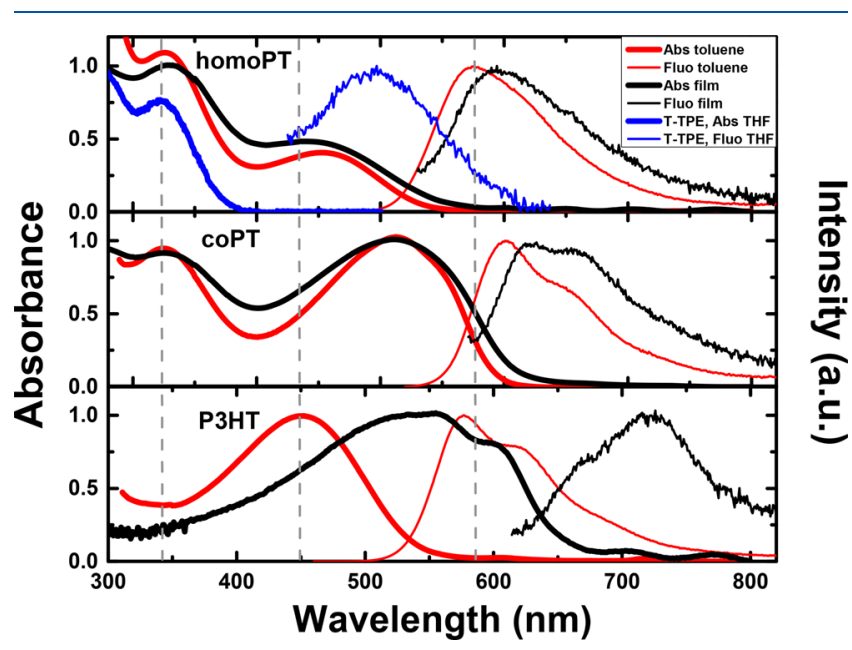

Figure 1. Room temperature normalized absorption and fluorescence emission spectra for the investigated polythiophenes in toluene solution and in thin films. characteristic of polythiophenes, thus showing that different potential energy curves (and geometries) are found in the ground state (chains torsionally disordered) and first singlet excited states (more planar conformations with a quinoidaltype character) of these compounds. ${ }^{21-24}$ The spectroscopic properties found for P3HT and homoPT closely resemble the absorption and fluorescence emission spectra found for heptathiophene $\left(\lambda_{\max }^{\mathrm{Abs}}=441 \mathrm{~nm}\right.$ and $\lambda_{\max }^{\text {Fluo }}=522$ and 560 $\mathrm{nm}^{14}$ ), thus showing that the size of the effectively conjugated segment for these polymers should be similar to this oligomer. Indeed, in general, it has been shown that the fully relaxed emissive segment length is $>7$ repeat units in P3HT. ${ }^{14,17,23}$

Noteworthy, and contrary to what was found for P3HT, is the appearance of an additional absorption band at higher energies $(325 \mathrm{~nm})$ for homoPT and coPT polymers; see Table 2 and Figure 1. Comparison with the absorption spectra of the model compound T-TPE (see Figure 1 and Scheme 3 ) shows that this additional spectroscopic feature of the polymers is also present in the model compound T-TPE and can be related to the TPE chromophore $\left(\lambda_{\max }^{\text {Abs }}=323 \mathrm{~nm}\right.$ and $\lambda_{\max }^{\text {Fluo }}=495 \mathrm{~nm}$ for T-TPE in tetrahydrofuran solution). In addition, when comparison is made between the lowest energy absorption band of the $\mathrm{P} 3 \mathrm{HT}$ and the poly(tetraphenylethene-thiophenes), derivatives a red-shift of $\sim 5 \mathrm{~nm}$ was observed for homoPT while a more significant red-shift, $\sim 63 \mathrm{~nm}$, was observed for coPT (Table 2 and Figure 1). The higher redshift found for coPT when compared to P3HT (and homoPT) can be ascribed to the bulkier TPE side units (and substitution degree) induced backbone planarization promoting a stronger electronic coupling between the chromophoric repeating units. $^{25-27}$ Indeed, as previously reported for phenylsubstituted polythiophenes, ${ }^{27}$ for homoPT the polymer backbone should adopt a more twisted conformation due to the high substitution density with TPE side groups at each thiophene unit, whereas for coPT only each second thiophene unit carries a TPE side chain, resulting in a more planar polythiophene.

In the solution emission spectra, no significant changes were found in the fluorescence wavelength maxima for the homoPT when compared to the $\mathrm{P} 3 \mathrm{HT}$ polymer (although a decrease in the vibronic structure was observed). For coPT, instead, in agreement to what was observed in the absorption spectra, a significant $\sim 25 \mathrm{~nm}$ red-shift of the emission maximum was observed. Within experimental error the spectroscopic properties for P3HT and homoPT can be considered as identical, thus showing the existence of similar effective conjugation lengths ( $\pi$-delocalization degree) ${ }^{25}$

The obtained photophysical parameters (quantum yields, lifetimes and rate constants) in solution and in the solid state (thin films) are presented in Table 2. In solution (toluene and tetrahydrofuran), the fluorescence quantum yields $\left(\phi_{\mathrm{F}}\right)$ were found to be in the $0.13-0.22$ range (see Table 2 ). However, the obtained $\phi_{\mathrm{F}}$ values should be considered to remain, within experimental error, constant for these derivatives. In agreement to what was previously mentioned, in which similar conjugation segments should be adopted in P3HT and the tetraphenylethene-substituted homoPT, the consistency in the $\phi_{\mathrm{F}}$ values also suggests that similar properties are present in solution. The room temperature fluorescence quantum yields found for homoPT and coPT are in agreement with the values reported for phenyl-substituted polythiophenes (with substituents on every thiophene unit) and polybithiophenes. ${ }^{27}$ For P3HT, the $\phi_{\mathrm{F}}$ value found is in good agreement with the values 
Table 2. Spectroscopic (Absorption and Emission Maxima) and Photophysical Data (Fluorescence, $\phi_{\mathrm{F}}$, and Internal Conversion, $\phi_{\mathrm{IC}}$, Quantum Yields, and Rate Constants Together with the Sensitized Singlet Oxygen Formation Quantum Yields, $\phi_{\Delta}$, and Fluorescence Lifetimes, $\tau_{\mathrm{F}}$ ) for the Investigated Polythiophenes in Toluene Solution and in the Solid State (Thin Films) at $293 \mathrm{~K}$

\begin{tabular}{|c|c|c|c|c|c|c|c|c|c|c|c|c|c|}
\hline \multirow[b]{2}{*}{ compd } & \multicolumn{2}{|c|}{$293 \mathrm{~K}$} & \multicolumn{2}{|c|}{ film } & \multirow{2}{*}{$\frac{293 \mathrm{~K}}{\phi_{\mathrm{F}}}$} & \multirow{2}{*}{$\frac{\text { film }}{\phi_{\mathrm{F}}}$} & \multicolumn{7}{|c|}{$293 \mathrm{~K}$} \\
\hline & $\overline{\lambda_{\max }^{\mathrm{Abs}}(\mathrm{nm})}$ & $\lambda_{\max }^{\text {Fluo }}(\mathrm{nm})$ & $\lambda_{\max }^{\mathrm{Abs}}(\mathrm{nm})$ & $\lambda_{\max }^{\text {Fluo }}(\mathrm{nm})$ & & & $\overline{\tau_{\mathrm{F}}(\mathrm{ps})^{a}}$ & $\phi_{\mathrm{IC}}^{b}$ & $\phi_{\Delta}$ & $k_{\mathrm{F}}\left(\mathrm{ns}^{-1}\right)$ & $k_{\mathrm{IC}}\left(\mathrm{ns}^{-1}\right)$ & $k_{\mathrm{ISC}}\left(\mathrm{ns}^{-1}\right)$ & $k_{\mathrm{NR}}\left(\mathrm{ns}^{-1}\right)$ \\
\hline P3HT & 451 & 577 & 555 & 725 & 0.19 & 0.009 & 524 & 0.54 & 0.27 & 0.363 & 1.03 & 0.745 & 1.55 \\
\hline homoPT & 455 & 575 & 445 & 595 & 0.13 & 0.08 & 528 & 0.82 & 0.052 & 0.246 & 1.55 & 0.211 & 1.65 \\
\hline coPT & 513 & 600 & 513 & 620 & 0.22 & 0.03 & 715 & 0.68 & 0.10 & 0.308 & 0.951 & 0.325 & 1.09 \\
\hline
\end{tabular}

${ }^{a}$ Major component, associated with the decay of the relaxed polymer, of a multiexponential decay; see text for further details. ${ }^{b}$ Calculated assuming $\phi_{\Delta} \approx \phi_{\mathrm{T}}$.
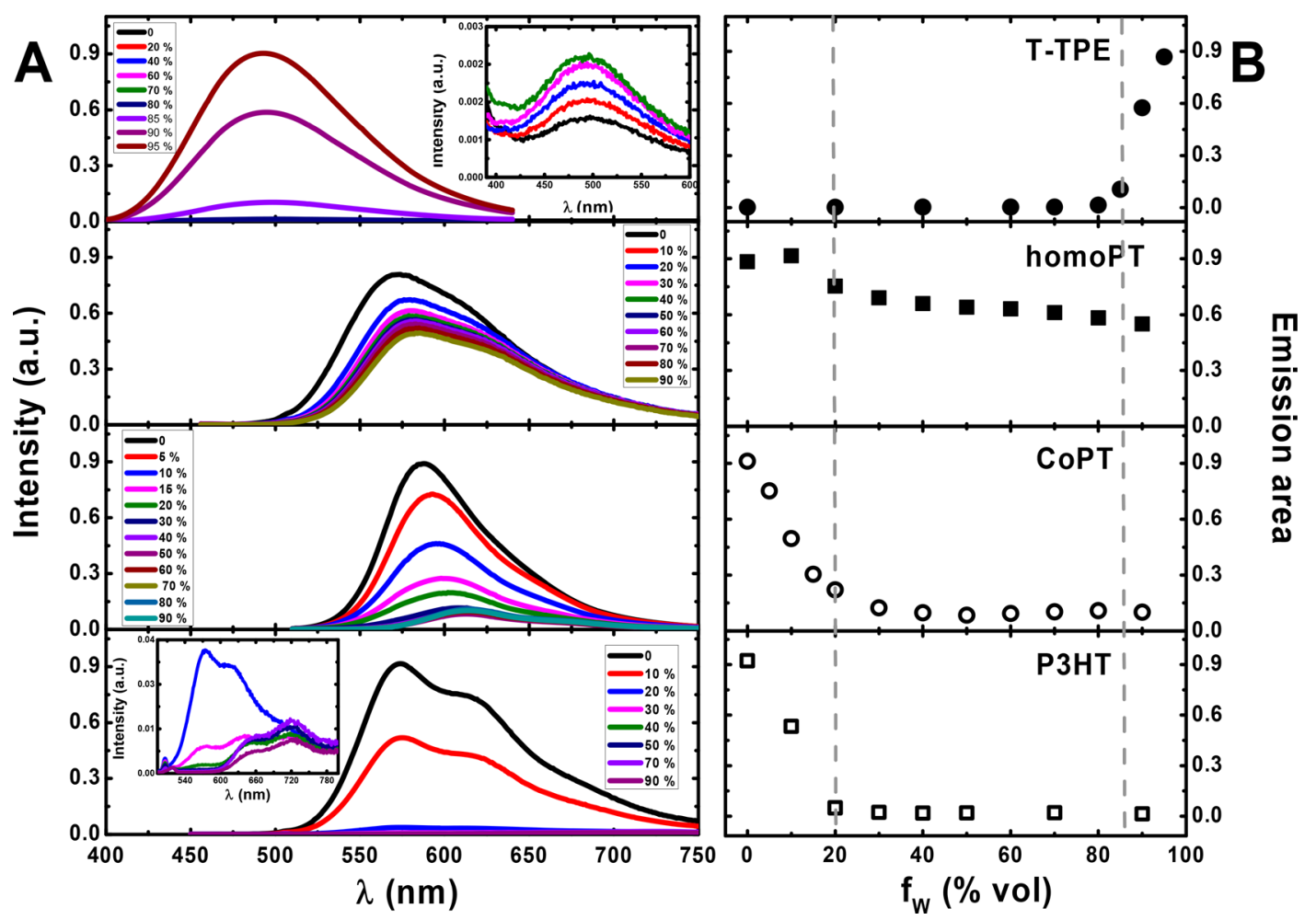

Figure 2. (A) Fluorescence emission spectra and (b) correlation of emission area with increasing water fractions $\left(f_{\mathrm{w}}\right)$ in THF/water mixtures for the polythiophenes, homoPT, coPT, and P3HT, together with the model compound T-TPE.

previously found for a regioregular polymer, RR-P3HT, with $\bar{M}_{\mathrm{w}}=55 \mathrm{kDa}$ in chlorobenzene solution, $\phi_{\mathrm{F}}=0.33$. $^{28}$

Upon going to the solid state (thin films), it was found that the absorption and emission spectra of homoPT and coPT closely resemble the spectroscopic features observed in solution (although with a $\sim 20 \mathrm{~nm}$ red-shift in the emission spectra; see Table 2). As described elsewhere, ${ }^{27,29,30}$ the steric hindrance of the TPE bulky groups and increased rigidity prevent the polymer chains from packing (by increasing the separation distance between polymer chains) and lead to similar spectroscopic properties in solution and in the solid state. On the contrary, for the investigated P3HT $\left(\bar{M}_{w}=18500\right.$ $\mathrm{g} \mathrm{mol}^{-1}$ ) and as previously reported for polythiophenes (including P3HT with different $\bar{M}_{\mathrm{w}}$ values), significant differences were seen when going from solution to solid films. Indeed, for the investigated P3HT, the unstructured absorption found in toluene solution is replaced by a structured and significantly red-shifted $(\sim 104 \mathrm{~nm})$ absorption band in thin films (Figure 1). Moreover, the emission band in the solid film is also significantly red-shifted $(\sim 148 \mathrm{~nm})$ and the intensity progression of the emission peaks was found to be opposite to that seen in solution with the low energy peak being more intense than the high energy peak. This behavior together with the decrease in the $\phi_{\mathrm{F}}$ value when going from solution to the solid state ( 0.19 vs. 0.009 , respectively) has been previously attributed to the emission from interchain (aggregate) excited states in P3HT films, where symmetry considerations reduce the intensity of the high energy emission. $^{28,31}$ Similar behavior was obtained at low temperatures where an additional resolved emission band appears at longer wavelengths for temperatures below $-20^{\circ} \mathrm{C}$ (see Figure $\mathrm{S} 1$ in the Supporting Information (SI)). The nature of this additional band may be questioned regarding its origin: it is (i) a consequence of a higher level of $\pi$-delocalization within the polymer or (ii) a consequence of aggregate formation; or of the two as suggested elsewhere for $\mathrm{P} 3 \mathrm{HT}$ where aggregation is found to be responsible for partial planarization with a simultaneous electronic coupling between segments of neighboring polymer chains. ${ }^{3}$ 
An opposite behavior was found for homo- and coPT where no additional bands (in the emission and excitation spectra, see Figures S2 and S3 in SI) appeared upon decreasing temperature that could be attributed to the formation of aggregates. Moreover, for the poly(tetraphenylethene-thiophenes), homoPT and coPT, although similar spectral features were found in solution and in thin films (which could indicate that similar excited states properties are found in these mediums), upon going to the solid state, there is a decrease in $\phi_{\mathrm{F}}$ values which is more pronounced for coPT $\left(\phi_{\mathrm{F}}=0.22\right.$ in toluene vs. $\phi_{\mathrm{F}}=0.03$ in the solid film $)$ than for homoPT $(0.13$ vs. 0.08); see Table 2. These results suggest that for homoPT, with a TPE substituent in each thiophene unit, the higher steric hindrance reduces the planarization of the main chain in the film and thus the aggregation caused quenching process is less efficient than for other polythiophene counterparts. Indeed, the more planar conformation expected for the coPT polythiophene when compared to homoPT allows intermolecular interactions that can favor aggregation caused quenching. Thus, to further explore this effect, we present a more detailed analysis of the emission behavior of these polymers and the T-TPE model compound in mixtures of good and poor solvents, i.e., in which the incremental formation of aggregates can be promoted.

Aggregation Caused Quenching (ACQ) Studies. To evaluate the occurrence of aggregation caused quenching (ACQ) in the tetraphenylethene-substituted polythiophenes, the fluorescence behavior in THF/water mixtures was investigated with different water fractions $\left(f_{\mathrm{w}}\right.$, the volume percentage of water in THF/water mixtures). Figure 2 displays the fluorescence emission spectra of the polymers homoPT, coPT, and P3HT, together with the data for the tetraphenylethene-thiophene model compound T-TPE. For T-TPE, in agreement with what was previously reported for $\mathrm{TPE}^{10}$ substituted thiophene monomers, ${ }^{32}$ a clear enhancement of the emission band centered at $495 \mathrm{~nm}$ was observed for mixtures with a water fraction $\geq 90 \%$ (Figure 2), thus showing that for T-TPE the aggregation induced emission (AIE) effect is observed.

For the polymers, a red-shift of the emission bands was found on going from THF to the 10:90 (v/v) THF/water mixture (where the highest degree of aggregation is expected); see Figure 2. Again, the most pronounced shift was observed for P3HT, displaying an absorption maximum red-shift of $\sim 152 \mathrm{~nm}$ vs $28 \mathrm{~nm}$ for coPT vs $10 \mathrm{~nm}$ for homoPT.

For the polymers coPT and P3HT, the aggregation caused emission quenching is clearly the dominant effect starting from very low water fractions (see Figure 2). While for homoPT, in agreement with the negligible decrease in the $\phi_{\mathrm{F}}$ values going from solution to solid films (from 0.13 to 0.08 , respectively, Table 2), only a minor, gradual decrease (if compared to coPT, see Figure 2) in emission intensity is observed up to the mixture with the highest water content. Thus, contrary to what is observed for the coPT polymer, the characteristic thiophenetetraphenylethene monomeric unit of the homoPT polymer restricts (although not completely) the formation of nonemissive aggregates. Indeed, it was expected that the conjugation of the TPE AIEgen with the polythiophenes could result, similarly to the monomeric T-TPE, in both AIE (based on the RIR effect) and intense aggregation-state emission. However, as described elsewhere ${ }^{27}$ for other polythiophenes with a similar bulk substituent pattern, for homoPT, the reduced intra- and intermolecular quenching is attributed to the twisted polymer conformation promoted by the high substitution density at each thiophene unit and steric hindrance of the TPE groups, which hinders close molecular packing.

On the other hand, for coPT, only each second thiophene unit carries a TPE side chains, resulting in a more planar polythiophene backbone thus allowing intermolecular interactions (aggregation) accompanied by red-shifts of the absorption and emission bands. Moreover, our results also suggest that the ability for conjugated polymer chromophores to interact depends on their mutual accessibility of the chains, as a prerequisite for an interaction with neighboring chromophores. It can also be noticed that the close similarity of the shapes and positions of the emission bands of these polymers in a poor solvent (e.g., the 10:90 THF/water mixture $)$ and in solid films $\left(\lambda_{\max }=615-620 \mathrm{~nm}\right.$ for coPT and $725 \mathrm{~nm}$ for P3HT; see Figures 1 and 2 and Table 2) indicate a similar environment of the emitting species in the two mediums.

Time Resolved Fluorescence. The fluorescence decays for the investigated polythiophenes were collected in toluene solution (as a function of the emission wavelength and at different temperatures) and in thin films (see Figure 3 and Table 3).

From the global analysis of the decays, i.e., simultaneous analysis of the decays at the three collected wavelengths), it was seen that, in toluene solution, in general, the decays were best fitted with a triexponential decay law with decay time values in the $13-65,82-309$, and $524-715$ ps ranges (see Table 3).

As listed in Table 3, the pre-exponential factors are positive when the decays are collected in the onset of the emission band, although when the emission decays are collected at lower energies (on the tail of the emission band), a negative amplitude (rise-time), associated with the shortest decay time (and in the case of P3HT also for the intermediate decay), is present. As described elsewhere, for different types of families of organic conjugated polymers and oligomers, the presence of a fast component, which appears as a decay time in the high energy part of the emission spectra and as a rise-time for low energies, is in general assigned to fast relaxation processes (excitation energy migration or conformational relaxation) in the excited state. ${ }^{15,21,33-37}$ For all the polymers, the preexponential factors associated with the slower decay time, $\tau_{1}$ (see Table 3), increases when going from the onset to the tail of the emission band and is assigned to the relaxed and more stable (lower energy) conformation of the polymers. An opposite behavior was found for the fastest decay component (and intermediary decay component in the P3HT case) where, respectively, a decrease and appearance of negative preexponential values were found when going to lower energies in the emission band. This indicates that more relaxed (lower energy) species of the polymers are being formed in the excited state at the expense of the nonrelaxed structures (associated with the shortest decay time for homo- and coPT, and for the two shortest decay components in the case of P3HT).

Additional information on the photophysical behavior of these compounds was obtained from the temperature dependence of the fluorescence decays since conformational relaxation is a temperature and solvent (viscosity) activated process. ${ }^{34,38}$ Within the temperature range studied, the longest decay component, $\tau_{1}$, for the investigated polythiophenes was found to be temperature independent (see Tables S1-S3 in the SI). 

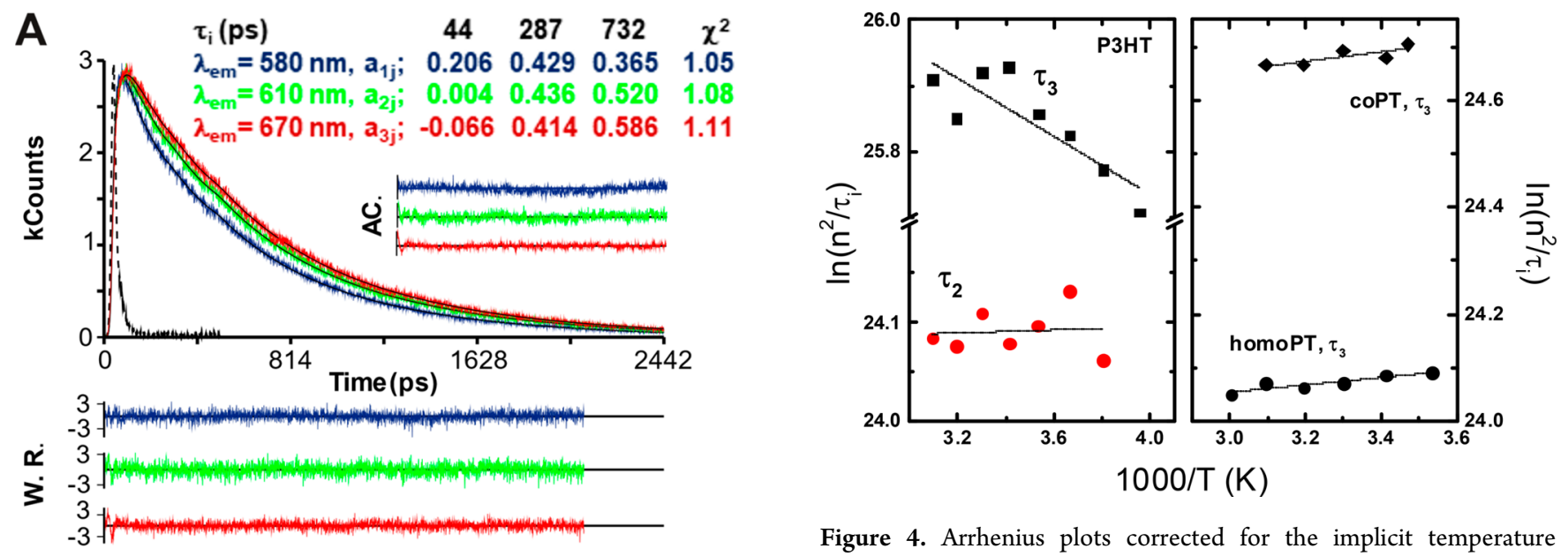

B
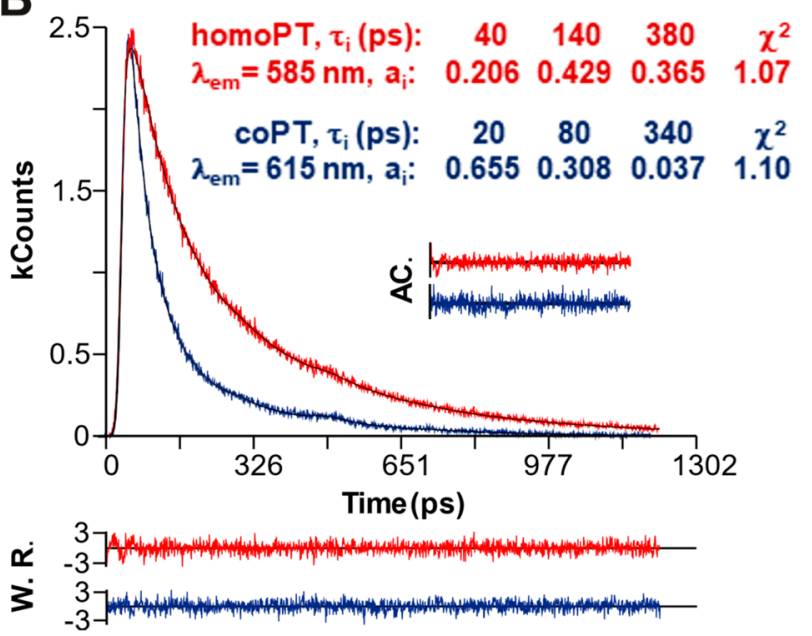

Figure 3. Room-temperature fluorescence decays for (A) coPT in toluene solution and (B) homo- and coPT in the solid state (thin films). For a better judgment of the quality of the fit, weighted residuals (W.R.), autocorrelation functions (AC.), and $\chi^{2}$ values are also presented. The dashed line in the decay of (A) corresponds to the instrumental response function.

However, the Arrhenius plots in Figure 4 of the reciprocal of the shortest lifetimes (associated with a negative preexponential value) and of the intermediate lifetime, $\tau_{2}$, in the case of P3HT show: (i) for homo- and coPT, the shortest decay time remains unchanged, at $\sim 65$ and $\sim 44$ ps,

Figure 4. Arrhenius plots corrected for the implicit temperature dependence of the energy-transfer rate constant on the solvent refractive index, $n_{\mathrm{D}}$, of the reciprocal of the shortest decays times $\left(\tau_{3}\right.$ and $\tau_{2}$ ) collected by time-correlated single photon counting.

respectively, thus pointing out the absence of conformational relaxation in these polymers; (ii) for P3HT $\left(\bar{M}_{\mathrm{w}}=18500 \mathrm{~g}\right.$ $\left.\mathrm{mol}^{-1}\right)$, the shortest decay time $\left(\tau_{3}\right)$ was found to be temperature dependent (Figure 4), while, within the experimental error, the intermediary decay component remains approximately constant, $\sim 80$ ps (see Figure 4 and Table $S 1$ in the SI). Since $\tau_{3}$ for P3HT is associated with an activated process (i.e., conformational relaxation), from the slope of the Arrhenius plot in Figure 4, it was possible to estimate the associated activation energy, $E_{\mathrm{a}}^{\mathrm{CR}}=1.8 \mathrm{~kJ} \mathrm{~mol}^{-1}$. Moreover, conformational relaxation rate constants in the $(5.90-7.71) \times$ $10^{10} \mathrm{~s}^{-1}$ range were found for P3HT in toluene when going from 253 to $323 \mathrm{~K}$ (see the Supporting Information for further details).

Summarizing at this stage, we can conclude that for homoand coPT excited state conformational relaxation is absent, with the energy migration/transfer being the dominant excited state decay process, occurring on time scales between 33 and 65 ps. For P3HT, it was found that the excited state decay involves competition between conformational relaxation (associated with decay times in the $12.8-15.4$ ps range) and energy migration ( $~ 80 \mathrm{ps})$. Although, for P3HT with higher average molecular weight $\left(\bar{M}_{w}=87 \mathrm{kDa}\right)$ it was suggested that the energy transfer process accounts for the two shortest decay components found by time-resolved fluorescence, ${ }^{34}$ other

Table 3. Room Temperature Fluorescence Decays Times and Pre-exponential Factors $\left(a_{\mathrm{ij}}\right)$ from the Best Fit to the Fluorescence Decays of the Investigated Polythiophenes in Toluene Solution and in the Solid State (Thin Films)

\begin{tabular}{|c|c|c|c|c|c|c|c|c|}
\hline compd & & $\tau_{3}(\mathrm{ps})$ & $\tau_{2}(\mathrm{ps})$ & $\tau_{1}(\mathrm{ps})$ & $\lambda_{\mathrm{em}}(\mathrm{nm})$ & $a_{\mathrm{i} 3}$ & $a_{\mathrm{i} 2}$ & $a_{\mathrm{i} 1}$ \\
\hline \multirow[t]{4}{*}{ P3HT } & toluene & 13 & 82 & 524 & 530 & 0.750 & 0.150 & 0.100 \\
\hline & & & & & 570 & -0.203 & 0.103 & 0.897 \\
\hline & & & & & 680 & -0.838 & -0.103 & 1.0 \\
\hline & film & 25 & 76 & 281 & 725 & 0.669 & 0.297 & 0.034 \\
\hline \multirow[t]{4}{*}{ homoPT } & toluene & 65 & 233 & 528 & 540 & 0.471 & 0.473 & 0.057 \\
\hline & & & & & 575 & 0.023 & 0.702 & 0.275 \\
\hline & & & & & 640 & -0.182 & 0.609 & 0.391 \\
\hline & film & 40 & 140 & 380 & 585 & 0.324 & 0.481 & 0.195 \\
\hline \multirow[t]{4}{*}{ coPT } & toluene & 44 & 287 & 732 & 580 & 0.206 & 0.429 & 0.365 \\
\hline & & & & & 610 & 0.044 & 0.436 & 0.520 \\
\hline & & & & & 670 & -0.066 & 0.414 & 0.586 \\
\hline & film & 20 & 80 & 340 & 615 & 0.655 & 0.308 & 0.037 \\
\hline
\end{tabular}


studies reported that for P3HT samples of increasing molecular weight (ranging from an average of 39 monomers to an average of 168 monomers) both conformational relaxation and energy migration are present. ${ }^{23}$

Nevertheless, for homoPT and coPT, the sterically bulky TPE pendant groups hinders fast torsional molecular movements of the polymer backbone, thus explaining the absence of conformational relaxation for the homoPT and coPT polythiophenes. $^{35}$

To further understand the nature of the different dominant decay mechanism observed for P3HT in comparison with homoPT and coPT, the time-resolved behavior of the regioregular poly(3-dodecylthiophene-2,5-diyl), P3DDT, in toluene solution as a function of temperature was investigated. Again, three decay components (with decay times of 30, 190, and 520 ps at $293 \mathrm{~K}$ ) were found when collecting the decay profiles along the emission band of the polymer, (see Table S4; and for the P3DDT detailed study and discussion, see the Supporting Information). Once more, the intermediate decay component was associated with an activated process, i.e., conformational relaxation, with an $E_{\mathrm{a}}^{\mathrm{CR}}$ value of $7.9 \mathrm{~kJ} \mathrm{~mol}^{-1}$ (Figure S4). The higher activation energy found for P3DDT when compared to $\mathrm{P} 3 \mathrm{HT}$, together with the increase in the lifetime values associated with this process when going from P3HT to P3DDT, shows that the size of the lateral side chains (from -hexyl to -dodecyl with higher solute solvent interactions) slows down the conformational relaxation process. $^{35}$

Singlet Oxygen Measurements. Singlet oxygen sensitization quantum yields were measured by direct measurement of the NIR singlet oxygen phosphorescence emission at 1275 $\mathrm{nm}$ of aerated toluene solutions of the polythiophenes after laser irradiation at $355 \mathrm{~nm}$. The $\phi_{\Delta}$ values were found to increase when going from homoPT, $\phi_{\Delta}=0.052$, to P3HT, $\phi_{\Delta}$ $=0.27$; see Table 2 . Assuming unitary singlet oxygen sensitization efficiency, i.e., $\phi_{\mathrm{T}} \cong \phi_{\Delta}$, it was possible to obtain the overall excited state deactivation rate constants (Table 2 ). From the photophysical parameters obtained in diluted toluene solution (i.e., involving in principle isolated polymer chains) and in the solid state (Table 2), we can conclude that in general the radiationless processes $\left(\phi_{\mathrm{IC}}+\phi_{\mathrm{ISC}}\right)$ are the dominant excited-state deactivation channels for the polymers studied.

\section{CONCLUSIONS}

In order to suppress the strong intermolecular interactions, the well-known AIE luminogen, TPE, was successfully integrated in two new polythiophenes with different contents of TPE side chains. For a monomeric model compound T-TPE, it was found that it displays significant aggregation induced emission in THF/water mixtures. An opposite behavior was observed for the polymers coPT and P3HT, where aggregation caused emission quenching is the dominant observed effect beginning from very low water fractions. For homoPT, the aggregation caused emission quenching is significantly reduced as seen by the small decrease in the fluorescence quantum yields going from solution to the solid state and by the small decrease in emission intensity observed in THF/water mixtures up to high water contents of $90 \%$ (transition from good to poor solvents). This shows that a higher degree of TPE substituents (as in homoPT) restricts the formation of nonemissive aggregates.

From the time-resolved fluorescence study as a function of temperature, it was found that for the TPE-substituted polythiophenes excited state energy migration along the polymer backbone occurs. Again, the absence of conformational relaxation for homoPT and coPT can be attributed to the restriction of torsional movements promoted by the bulky TPE substituents.

Although the AIE strategy based on the RIR has produced numerous systems with high emission in the aggregate state for the TPE substituted polythiophenes, only restriction of the formation of nonemissive aggregates was observed. Nevertheless, this contributes to a better understanding of the phenomena.

\section{ASSOCIATED CONTENT}

\section{SI Supporting Information}

The Supporting Information is available free of charge at https://pubs.acs.org/doi/10.1021/acs.jpcc.9b10908.

Steady-state and time-resolved fluorescence data collected as a function of temperature for the investigated polythiophenes; time-resolved behavior of the regioregular poly(3-dodecylthiophene-2,5-diyl), P3DDT, in toluene solution as a function of temperature, together with the Arrhenius and $\log -\log$ plots of $\mathrm{k} C \mathrm{CR} v s$. viscosity (derived from the Stokes-Einstein relation) and activation energies for the conformational relaxation process in the P3HT and P3DDT are presented and discussed considering solutesolvent interactions (PDF)

\section{AUTHOR INFORMATION}

\section{Corresponding Author}

João Pina - University of Coimbra, Coimbra Chemistry Centre, Department of Chemistry, Rua Larga 3004-535 Coimbra, Portugal; @ orcid.org/0000-0003-1848-1167; Email: jpina@ qui.uc.pt

\section{Authors}

Ana Clara B. Rodrigues - University of Coimbra, Coimbra Chemistry Centre, Department of Chemistry, Rua Larga 3004 535 Coimbra, Portugal; (i) orcid.org/0000-0002-5128-0204

Mohamed Alnady - University of Coimbra, Coimbra Chemistry Centre, Department of Chemistry, Rua Larga 3004-535 Coimbra, Portugal

Wenyue Dong - Macromolecular Chemistry Group (buwmakro) and Institute for Polymer Technology, Bergische Universitat Wuppertal, D-42097 Wuppertal, Germany

Ullrich Scherf - Macromolecular Chemistry Group (buwmakro) and Wuppertal Institute for Smart Materials and Systems, Bergische Universitat Wuppertal, D-42097 Wuppertal, Germany; ○ orcid.org/0000-0001-8368-4919

J. Sérgio Seixas de Melo - University of Coimbra, Coimbra Chemistry Centre, Department of Chemistry, Rua Larga 3004535 Coimbra, Portugal; 이이이.org/0000-0001-9708-5079

Complete contact information is available at:

https://pubs.acs.org/10.1021/acs.jpcc.9b10908

\section{Notes}

The authors declare no competing financial interest.

\section{ACKNOWLEDGMENTS}

This work was supported by Project "Hylight" (no. 031625) 02/SAICT/2017, PTDC/QUI-QFI/31625/2017, which is funded by the Portuguese Science Foundation and Compete Centro 2020. We acknowledge funding by Fundo Europeu de 
Desenvolvimento Regional (FEDER) through Programa Operacional Factores de Competitividade (COMPETE) and Project ROTEIRO/0152/2013. The Coimbra Chemistry Centre is supported by the Fundação para a Ciência e a Tecnologia (FCT), Portuguese Agency for Scientific Research, through the projects UIDB/QUI/00313/2020 and UIDP/ QUI/00313/2020. A.C.B.R. acknowledges Project No. 22124LLPT-Laserlab-Portugal (ref: CENTRO-01-0145-FEDER000014) for a postdoctoral grant. J.P. and J.S.S.M. acknowledge Project "SunStorage - Harvesting and storage of solar energy" for financial support, reference POCI-01-0145FEDER-016387, funded by European Regional Development Fund (ERDF), through COMPETE 2020-Operational Programme for Competitiveness and Internationalization (OPCI), and by national funds, through FCT. The research leading to these results has received funding from Laserlab-Europe (Grant Agreement No. 284464, EC's Seventh Framework Programme).

\section{REFERENCES}

(1) Wang, H.; Huang, J.; Uddin, M. A.; Liu, B.; Chen, P.; Shi, S. B.; Tang, Y. M.; Xing, G. C.; Zhang, S. M.; Woo, H. Y.; et al. CyanoSubstituted Head-to-Head Polythiophenes: Enabling High-Performance n-Type Organic Thin-Film Transistors. ACS Appl. Mater. Interfaces 2019, 11, 10089-10098.

(2) Hamdast, A.; Agbolaghi, S.; Zeighami, M.; Beygi-Khosrowshahi, Y.; Sarvari, R. Butterfly nanostructures via regioregularly grafted multiwalled carbon nanotubes and poly(3-hexylthiophene) to improve photovoltaic characteristics. Polym. Int. 2019, 68, 335-343.

(3) Raithel, D.; Baderschneider, S.; de Queiroz, T. B.; Lohwasser, R.; Köhler, J.; Thelakkat, M.; Kümmel, S.; Hildner, R. Emitting Species of Poly(3-hexylthiophene): From Single, Isolated Chains to Bulk. Macromolecules 2016, 49, 9553-9560.

(4) Würthner, F.; Kaiser, T. E.; Saha-Möller, C. R. J-Aggregates: From Serendipitous Discovery to Supramolecular Engineering of Functional Dye Materials. Angew. Chem., Int. Ed. 2011, 50, 33763410.

(5) Hong, Y.; Lam, J. W. Y.; Tang, B. Z. Aggregation-Induced Emission. Chem. Soc. Rev. 2011, 40, 5361-5388.

(6) Hu, R.; Kang, Y.; Tang, B. Z. Recent Advances in AIE Polymers. Polym. J. 2016, 48, 359-370.

(7) Qiu, Z. J.; Liu, X. L.; Lam, J. W. Y.; Tang, B. Z. The Marriage of Aggregation-Induced Emission with Polymer Science. Macromol. Rapid Commun. 2019, 40, 1800568.

(8) Rodrigues, A. C. B.; Pina, J.; Dong, W.; Forster, M.; Scherf, U.; Seixas de Melo, J. S. Aggregation-Induced Emission in PhenothiazineTPE and -TPAN Polymers. Macromolecules 2018, 51, 8501-8512.

(9) Zhao, Z.; Lam, J. W. Y.; Tang, B. Z. Tetraphenylethene: A Versatile AIE Building Block for the Construction of Efficient Luminescent Materials for Organic Light-Emitting Diodes. J. Mater. Chem. 2012, 22, 23726-23740.

(10) Zhao, Z.; W. Y. Lam, J.; Zhong Tang, B. Aggregation-Induced Emission of Tetraarylethene Luminogens. Curr. Org. Chem. 2010, 14, 2109-2132.

(11) Turro, N. Modern Molecular Photochemistry; University Science Books: Sausalito, CA, 1991.

(12) Mei, J.; Leung, N. L. C.; Kwok, R. T. K.; Lam, J. W. Y.; Tang, B. Z. Aggregation-Induced Emission: Together We Shine, United We Soar! Chem. Rev. 2015, 115, 11718-11940.

(13) Montalti, M.; Credi, A.; Prodi, L.; Gandolfi, M. T. Handbook of Photochemistry, Third ed.; CRC Press: Boca Raton, FL, 2006.

(14) Becker, R. S.; Seixas de Melo, J.; Maçanita, A. L.; Elisei, F. Comprehensive Evaluation of the Absorption, Photophysical, Energy Transfer, Structural, and Theoretical Properties of alpha-Oligothiophenes With One to Seven Rings. J. Phys. Chem. 1996, 100, 1868318695.
(15) Pina, J.; Seixas de Melo, J.; Burrows, H. D.; Maçanita, A. L.; Galbrecht, F.; Bunnagel, T.; Scherf, U. Alternating BinaphthylThiophene Copolymers: Synthesis, Spectroscopy, and Photophysics and Their Relevance to the Question of Energy Migration versus Conformational Relaxation. Macromolecules 2009, 42, 1710-1719.

(16) Striker, G.; Subramaniam, V.; Seidel, C. A. M.; Volkmer, A. Photochromicity and Fluorescence Lifetimes of Green Fluorescent Protein. J. Phys. Chem. B 1999, 103, 8612-8617.

(17) Seixas de Melo, J.; Silva, L. M.; Arnaut, L. G.; Becker, R. S. Singlet and Triplet Energies of alpha-Oligothiophenes: A Spectroscopic, Theoretical, and Photoacoustic Study: Extrapolation to Polythiophene. J. Chem. Phys. 1999, 111, 5427-5433.

(18) Flors, C.; Nonell, S. On the Phosphorescence of $1 \mathrm{H}$-phenalen1-one. Helv. Chim. Acta 2001, 84, 2533-2539.

(19) Scharsich, C.; Lohwasser, R. H.; Sommer, M.; Asawapirom, U.; Scherf, U.; Thelakkat, M.; Neher, D.; Kohler, A. Control of aggregate Formation in Poly(3-hexylthiophene) by Solvent, Molecular Weight, and Synthetic Method. J. Polym. Sci., Part B: Polym. Phys. 2012, 50, $442-453$.

(20) Koenen, J.-M.; Jung, S.; Patra, A.; Helfer, A.; Scherf, U. DyeTerminated, Hyperbranched Polytruxenes and Polytruxene-blockPolythiophene Multiblock Copolymers Made in an "AB2 + A" Approach. Adv. Mater. 2012, 24, 681-686.

(21) Dias, F. B.; Maçanita, A. L.; Seixas de Melo, J.; Burrows, H. D.; Guntner, R.; Scherf, U.; Monkman, A. P. Picosecond Conformational Relaxation of Singlet Excited Polyfluorene in Solution. J. Chem. Phys. 2003, 118, 7119-7126.

(22) Hintschich, S. I.; Dias, F. B.; Monkman, A. P. Dynamics of Conformational Relaxation in Photoexcited Oligofluorenes and Polyfluorene. Phys. Rev. B: Condens. Matter Mater. Phys. 2006, 74, 045210 .

(23) Wells, N. P.; Boudouris, B. W.; Hillmyer, M. A.; Blank, D. A. Intramolecular Exciton Relaxation and Migration Dynamics in Poly(3-hexylthiophene). J. Phys. Chem. C 2007, 111, 15404-15414.

(24) Yamamoto, T.; Komarudin, D.; Arai, M.; Lee, B. L.; Suganuma, H.; Asakawa, N.; Inoue, Y.; Kubota, K.; Sasaki, S.; Fukuda, T.; et al. Extensive Studies on pi-Stacking of Poly(3-alkylthiophene-2,5-diyl)s and Poly(4-alkylthiazole-2,5-diyl)s by Optical spectroscopy, NMR Analysis, Light Scattering Analysis, and X-ray Crystallography. J. Am. Chem. Soc. 1998, 120, 2047-2058.

(25) Raithel, D.; Simine, L.; Pickel, S.; Schotz, K.; Panzer, F.; Baderschneider, S.; Schiefer, D.; Lohwasser, R.; Kohler, J.; Thelakkat, M.; et al. Direct Observation of Backbone Planarization Via SideChain Alignment in Single Bulky-Substituted Polythiophenes. Proc. Natl. Acad. Sci. U. S. A. 2018, 115, 2699-2704.

(26) Aasmundtveit, K. E.; Samuelsen, E. J.; Mammo, W.; Svensson, M.; Andersson, M. R.; Pettersson, L. A. A.; Inganäs, O. Structural Ordering in Phenyl-Substituted Polythiophenes. Macromolecules 2000, 33, 5481-5489.

(27) Theander, M.; Inganäs, O.; Mammo, W.; Olinga, T.; Svensson, M.; Andersson, M. R. Photophysics of Substituted Polythiophenes. J. Phys. Chem. B 1999, 103, 7771-7780.

(28) Cook, S.; Furube, A.; Katoh, R. Analysis of the Excited States of Regioregular Polythiophene P3HT. Energy Environ. Sci. 2008, 1, 294299.

(29) Angiolini, L.; Brazzi, A.; Salatelli, E.; Van den Bergh, K.; Koeckelberghs, G. Polythiophene Diblock Copolymer with Different Solvent Affinities of the Side-Chain Substituents: Solvatochromism and Effect on the Electronic Conjugation. Macromol. Chem. Phys. 2013, 214, 934-942.

(30) Wang, L.; Feng, Q.; Wang, X.; Pei, M.; Xu, J.; Zhang, G. Synthesis and Properties of Two Novel Regioregular Conjugated Polythiophenes with Side-Chain Containing Different Substituted End Groups. Des. Monomers Polym. 2013, 16, 116-126.

(31) Clark, J.; Silva, C.; Friend, R. H.; Spano, F. C. Role of Intermolecular Coupling in the Photophysics of Disordered Organic Semiconductors: Aggregate Emission in Regioregular Polythiophene. Phys. Rev. Lett. 2007, 98, 206406. 
(32) Li, J.; Shan, T.; Yao, M.; Gao, Y.; Han, X.; Yang, B.; Lu, P. The Effect of Different Binding Sites on the Optical and Electronic Properties of Tetraphenylethylene-Substituted Thiophene Isomers. J. Mater. Chem. C 2017, 5, 2552-2558.

(33) Di Paolo, R. E.; Seixas de Melo, J.; Pina, J.; Burrows, H. D.; Morgado, J.; Maçanita, A. L. Conformational Relaxation of pPhenylenevinylene (PV) Trimers in Solution, Studied by Picosecond Time-Resolved Fluorescence. ChemPhysChem 2007, 8, 2657-2664.

(34) Ferreira, B.; da Silva, P. F.; Seixas de Melo, J. S.; Pina, J.; Maçanita, A. Excited-State Dynamics and Self-Organization of Poly(3hexylthiophene) (P3HT) in Solution and Thin Films. J. Phys. Chem. B 2012, 116, 2347-2355.

(35) Galvão, A. M.; Di Paolo, R. E.; Maçanita, A. L.; Naqvi, K. R. Model for Conformational Relaxation of Flexible Conjugated Polymers: Application to p-Phenylenevinylene Trimers in Nonpolar Solvents. ChemPhysChem 2013, 14, 583-590.

(36) Pina, J.; Seixas de Melo, J. S.; Eckert, A.; Scherf, U. Unusual Photophysical Properties of Conjugated, Alternating Indigo-Fluorene Copolymers. J. Mater. Chem. A 2015, 3, 6373-6382.

(37) Pina, J.; Alnady, M.; Eckert, A.; Scherf, U.; Seixas de Melo, J. S. Alternating donor-Acceptor Indigo-Cyclopentadithiophene Copolymers: Competition Between Excited State Conformational Relaxation, Energy Transfer and Excited State Proton Transfer. Mater. Chem. Front. 2018, 2, 281-290.

(38) Maçanita, A. L.; Zachariasse, K. A. Viscosity Dependence of Intramolecular Excimer Formation with 1,5-Bis(1-pyrenylcarboxy)pentane in Alkane Solvents as a Function of Temperature. J. Phys. Chem. A 2011, 115, 3183-3195. 